\title{
ENRIQUECIMENTO FUNCIONAL DE CARNES E PRODUTOS CÁRNEOS
}

\author{
Bruno Henrique Figueiredo Saqueti ${ }^{1}$, Djéssica Tatiane Raspe², Luciana Alves da Silva ${ }^{3}$,
} Eloize da Silva Alves ${ }^{4}$, Denise de Moraes Batista da Silva ${ }^{5}$, Carla Adriana Ferrari Artilha 6

\author{
${ }^{1}$ Mestrando do Programa de Pós-Graduação em Ciência de Alimentos, Universidade Estadual de Maringá - UEM. \\ bruno_saqueti@outlook.com \\ ${ }^{2}$ Doutoranda do Programa de Pós-Graduação em Ciência de Alimentos, Universidade Estadual de Maringá - UEM. Bolsista CAPES. \\ djessicaraspe@hotmail.com \\ ${ }^{3}$ Doutoranda do Programa de Pós-Graduação em Ciência de Alimentos, Universidade Estadual de Maringá - UEM. \\ luciana.alves.engali@gmail.com \\ ${ }^{4}$ Mestranda do Programa de Pós-Graduação em Ciência de Alimentos, Universidade Estadual de Maringá - UEM. Bolsista CAPES. \\ eloizeetaus@gmail.com \\ ${ }^{5}$ Doutoranda do Programa de Pós-Graduação em Ciência de Alimentos, Universidade Estadual de Maringá - UEM. \\ denise_mbsilva@hotmail.com \\ ${ }^{6}$ Mestranda do Programa de Pós-Graduação em Ciência de Alimentos, Universidade Estadual de Maringá - UEM. Bolsista CAPES. \\ c.artilha@yahoo.com.br
}

\section{RESUMO}

Nos últimos anos, muita atenção tem sido dada ao desenvolvimento de carnes e produtos cárneos com funções fisiológicas para promover condições de saúde e prevenir o risco de doenças. Esta revisão concentra-se em estratégias para melhorar o valor funcional dos produtos cárneos e carnes. A melhoria do valor pode ser obtida pela adição de compostos funcionais, incluindo ácido linoléico conjugado, vitamina $E$, ácidos graxos $\omega 3$ e selênio em dietas animais para melhorar a produção animal, a composição da carcaça e a qualidade da carne fresca. Além disso, ingredientes funcionais como proteínas vegetais, fibras alimentares, ervas e especiarias podem ser incorporadas diretamente nos produtos cárneos durante o processamento para melhorar seu valor funcional. Compostos funcionais, especialmente peptídeos, também podem ser gerados a partir de produtos cárneos e carne durante o processamento, como fermentação, cura e revestimento comestível. Esta revisão discute ainda mais o status atual de pesquisa de desenvolvimento de produtos cárneos funcionais.

PALAVRAS-CHAVE: Alimentos funcionais; Compostos bioativos; Alimento natural; Alimento processado.

\section{INTRODUÇÃO}

Um alimento, para ser dito "funcional", deve conter um componente com efeito seletivo em uma ou várias funções do organismo cujos efeitos positivos possam ser justificados como funcionais (fisiológicos) ou mesmo saudáveis. Os três requisitos básicos a serem considerados como alimento funcional incluem 1) derivados de ingredientes naturais; 2) consumir como parte da dieta diária; e 3) envolvem na regulação de processos específicos para o ser humano, inclusive retardando o processo de envelhecimento, prevenindo o risco de doenças e melhorando a capacidade imunológica (JIMENEZ-COLMENERO; CARBALLO; COFRADES, 2001).

Carne e produtos à base de carne são fontes importantes de proteína, gordura, aminoácidos essenciais, minerais e vitaminas e outros nutrientes. Nos últimos anos, o consumidor exige carne e produtos de carne mais saudáveis com redução do nível de gordura, colesterol, diminuição do conteúdo de cloreto de sódio e nitrito, melhor composição do perfil de ácidos graxos e ingredientes incorporados que aumentam a saúde (BIESALSKI, 2005).

Enriquecimento de carne crua com compostos bioativos e os efeitos de substâncias à base de carne, como carnosina, anserina, L-carnitina, glutationa, taurina e creatina na saúde humana têm sido estudados extensivamente (ARIHARA, 2004). Durante 0 processamento da carne e produtos cárneos, muitos compostos funcionais podem ser gerados: muitos peptídeos produzidos a partir da fermentação mostraram benefícios fisiológicos para o ser humano (VERCRUYSSE; VAN CAMP; SMAGGHE, 2005). 
A aceitação do consumidor por alimentos funcionais varia muito, dependendo de suas origens sociais, econômicas, geográficas, políticas, culturais e étnicas (JIMENEZCOLMENERO; CARBALLO; COFRADES, 2001). O Japão foi o primeiro país que desenvolveu a ideia de alimentos funcionais e estabeleceu regulamentos para seu uso (KWAK; JUKES, 2001). Nos países europeus, o mercado deste tipo de alimento tem aumentado continuamente, enquanto que os consumidores dos países da Europa Central e do Norte, tem sido mais favorável a alimentos funcionais do que os dos países mediterrâneos, que preferem alimentos frescos e naturais (MENRAD, 2003).

Os alimentos funcionais são considerados uma nova tendência do mercado alimentício e, dentre as razões relacionadas ao crescente mercado de alimentos funcionais pode-se destacar: a perseguição desenfreada por dietas saudáveis valorizando demasiadamente um componente do alimento em detrimento a valorização do alimento como um todo; o reconhecimento pelas agências reguladoras dos benefícios dos alimentos funcionais para a saúde; a possibilidade de redução de custos no combate das doenças crônicas não transmissíveis (DCNTs), como obesidade, hipertensão arterial sistêmica, osteoporose, diabetes mellitus e câncer, por parte do Estado; e, por fim, para as indústrias que investiram em pesquisas e novas tecnologias. Os alimentos funcionais representam um nicho de mercado extremamente rentável, pois são produtos com alto valor agregado e com um marketing agressivo na busca pelo consumo (MARINS; ARAUJO; JACOB, 2011).

Nesse contexto, o objetivo do presente estudo foi revisar a literatura sobre elaboração de alimentos cárneos que contém ingredientes funcionais em sua constituição e formulação, tendo como intuito destacar a importância destes na alimentação humana.

\section{PRODUTOS CÁRNEOS FUNCIONAIS}

\subsection{ALIMENTAÇÃO ANIMAL COM INGREDIENTES FUNCIONAIS}

A influência da nutrição do animal nos tecidos adiposo e muscular tem sido estudada e reportada tanto na composição em ácidos graxos, vitaminas e minerais. Os interesses no ácido linoleico conjugado (CLA), em diversas pesquisas, devido numerosas propriedades fisiológicas e biológicas foram atribuídas, incluindo potencial antioxidante e anti-obesidade, anticarcinogênico, antiaterosclerótico, antidiabético, auxílio na proteção do sistema imunológico e contribuição para a formação óssea e corporal (LIMA JR et al., 2011).

Efeitos dietéticos visando aumentar o desempenho animal, melhorar a qualidade dos alimentos e fornecer produtos com altas quantidades de CLA também tem sido estudado e relatado. Paralelamente ao efeito benéfico, tem sido reportado que o CLA reduz significativamente a proporção de ácidos graxos insaturados e aumenta os ácidos graxos saturados em músculos bovinos (EGGERT et al., 2001) e em tecidos suínos (WIEGAND et al., 2002). Em pedaços de frango, o CLA dietético inserido na dieta dos animais também foi associado à geração de uma carne cozida mais dura, mais seca e escura, proporcional à redução de sua suculência (DU et al., 2003).

Por outro lado, tem sido amplamente relatado que a suplementação de vitamina $E$ na dieta de animais pode melhorar a qualidade da carne fresca e de produtos cárneos, limitando a oxidação de proteínas e lipídios e melhorando a cor da carne. Para a qualidade da carne fresca, a vitamina $E$ está possivelmente envolvida na regulação da conversão do músculo em carne, inibindo a oxidação proteica (CARNAGEY et al., 2008).

Já os ácidos graxos poliinsaturados de cadeia longa (AGPICL) são reconhecidos como constituintes essenciais para o crescimento e desenvolvimento normal em animais (LIMA JR et al., 2011). A principal fonte de AGPICL de cadeia longa é o peixe e outros frutos do mar, entanto, existem muitas outras fontes alimentares alternativas ricas em 
AGPICL de cadeia longa disponíveis, que incluem carne, leite e ovos de animais alimentados com dietas enriquecidas com $\omega 3$ (LIMA JR et al., 2011).

O selênio é um mineral essencial para humanos e animais porque está envolvido na regulação de várias funções fisiológicas como parte integrante das selenoproteínas. Em humanos, a deficiência de selênio está associada à diminuição de funções imunológicas, resultando em maior suscetibilidade ao câncer, doenças cardiovasculares, distrofia muscular, diabete, artrite, cataratas, acidente vascular cerebral, degeneração macular e outras doenças (MUELLER et al., 2009). Sendo uma fonte importante de selênio dietético para humanos, a concentração de selênio neste tipo de alimento varia dramaticamente entre países e regiões e sua incorporação através da suplementação dietética dos animais de corte pode ser uma excelente maneira de melhorar a ingestão deste mineral.

Cabe ressaltar que, uma vez que estes ingredientes estejam incorporados na carne, é de fundamental importância verificar cuidadosamente por meio de análise se estes ingredientes químicos e funcionais permanecem inalterados durante os processos de tecnologia de fabricação, durante o armazenamento por resfriamento e sobre sua vida de prateleira, bem como sua manutenção após as operações de preparação culinária. Isso garante que o potencial saudável dos produtos funcionalizados atinja o ponto de consumo.

\subsection{INCORPORAÇÃO DE INGREDIENTES FUNCIONAIS EM PRODUTOS DURANTE O PROCESSAMENTO}

Durante as últimas décadas, os aditivos têm sido amplamente utilizados em produtos cárneos para reduzir os custos dos produtos e melhorar sua funcionalidade. Estes aditivos incluem proteínas vegetais, fibras alimentares, ervas e especiarias que podem aumentar o valor nutricional e proporcionar benefícios para a saúde humana. As proteínas de soja são amplamente utilizadas em produtos cárneos nas formas de farinha e concentrados de soja para melhorar a capacidade de ligação de água e gordura, melhorar a estabilidade da emulsão, melhorar o conteúdo nutricional e aumentar rendimento (CHIN et al., 2000). Proteínas de soja são muito hidrofílicas e, portanto, podem ser incorporadas em produtos para reduzir a perda de cozimento, diminuindo a perda d'água durante o armazenamento refrigerado sem introduzir qualquer alteração no sabor, aroma, características de suculência, oxidação e estabilidade microbiológica (PORCELLA et al., 2001).

As proteínas do soro do leite mostraram excelentes propriedades nutricionais e funcionais em produtos cárneos com baixo teor de gordura (PEREZ-GAGO; KROCHTA, 2001), melhorando a estabilidade da emulsão, proporcionando melhores propriedades de cor, porém, resultando em menor mastigabilidade e elasticidade (YETIM; MULLER; EBER, 2001). Quando a proteína do soro de leite foi usada na massa de carne crua e cozida de aves, resultou em maior capacidade de retenção de água, melhores propriedades reológicas e perda de cozimento reduzida (HONGSPRABHAS; BARBUT, 1999).

A gordura é um constituinte importante para a nutrição humana como fonte de vitamina e ácidos graxos essenciais e fornece a maior parte da energia na dieta. Também pode contribuir para o sabor, maciez, suculência, aparência e textura dos produtos à base de carne (CAVESTANY et al., 1994). No entanto, o consumo excessivo de gordura está associado a várias doenças, incluindo obesidade, câncer e doenças coronarianas (ROTHSTEIN, 2006). Assim, a indústria da carne está tentando produzir produtos cárneos com baixo teor de gordura, sem comprometer as características sensoriais e de textura.

A fibra dietética é um dos ingredientes mais usados para fornecer a produtos à base de carne, baixo teor de gordura e aumento no teor de fibras. É definida como o 
remanescente da parte comestível das plantas, contendo carboidratos análogos que são resistentes à digestão e absorção no intestino delgado humano (PROSKY, 1999). O aumento da ingestão de fibras alimentares tem sido recomendado devido aos seus efeitos na redução do risco de câncer de cólon, diabetes, obesidade e doenças cardiovasculares (EASTWOOD, 1992). Sua adição em produtos cárneos tem sido reportada de forma benéfica para a estabilidade e qualidade dos produtos, aumentando a viscosidade e diminuindo o pH sem influenciar na perda de cozimento, conteúdo proteico, colágeno e avaliação sensorial (GRIGELMO-MIGUEL; ABADIAS-SEROS; MARTIN-BELLOSO, 1999).

A oxidação lipídica é a principal reação que deteriora o sabor, a cor, a textura e o valor nutricional dos alimentos. Vários antioxidantes sintéticos, como 0 hidroxitoluenobutilado (BHT), o hidroxianisolbutilado (BHA) e a butilhidroquinona terciária, têm sido usados para prevenir este efeito nos alimentos. Entretanto, os antioxidantes sintéticos não são completamente aceitos pelos consumidores devido a preocupações com a saúde. Portanto, alguns ingredientes naturais, incluindo ervas e especiarias, têm sido estudadas especialmente em países asiáticos como potenciais antioxidantes em carnes e em produtos cárneos (MCCARTHY et al., 2001).

Compostos de ervas e especiarias contêm muitos fitoquímicos que são fontes potenciais de antioxidantes naturais, incluindo diterpenos fenólicos, flavonóides, taninos e ácidos fenólicos (DAWIDOWICZ; WIANOWSKA; BARANIAK, 2006). Estes compostos possuem atividades antioxidante, antiinflamatória e anticancerígena. Nos sistemas alimentares, podem melhorar o sabor, retardar a deterioração induzida pela oxidação lipídica, inibir o crescimento de microrganismos e desempenhar um papel importante na diminuição do risco de algumas doenças (TANABE; YOSHIDA; TOMITA, 2002). Entre as especiarias, o cravo é relatado como tendo a mais forte capacidade antioxidante, seguida por pétalas de rosa, canela, noz-moscada, entre outros. Além disso, as especiarias têm capacidade antimicrobiana, principalmente devido aos compostos fenólicos (BAJPAI et al., 2008).

Neste contexto, podemos destacar o extrato de alecrim como conservante natural, uma vez que contém altos níveis de compostos fenólicos levando a sua grande atividade antioxidante. Tem sido relatado que os extratos de alecrim foram iguais ou mais efetivos que BHA/BHT em retardar os valores de TBARS em salsichas cruas e pré-cozidas durante o armazenamento refrigerado e congelado (SEBRANEK et al., 2004). A efetividade em retardar a oxidação lipídica e prevenir a perda de cor durante o armazenamento refrigerado também foi reportada (YU et al., 2002).

A alicina é conhecida como o principal ingrediente do alho, e tem sido reportada com atividades antimicrobianas contra bactérias gram-positivas e gram-negativas. Muitos estudos demonstraram que o extrato de alho foi eficaz na redução do crescimento de muitos patógenos, incluindo $S$. aureus, S. albus, S. typhi, E. coli, L. monocytogenes, $A$. niger, parasita Acari, Pseudomonas aeruginosa e Proteusmorganni (MAIDMENT; DEMBNY; HARDING, 1999). Em carnes frigorificadas de frango, o extrato aquoso de alho inibiu o crescimento de contaminantes microbianos, incluindo coliformes aeróbios, mesófilos e fecais facultativos na superfície de carcaças de frango (OLIVEIRA et al., 2005).

O orégano é uma especiaria mediterrânea tradicional e seu óleo essencial obtido através do processo de destilação a vapor contém mais de 30 compostos. Entre os compostos, o carvacrol e o timol constituem sua principal capacidade antioxidante (VEKIARI et al., 1993). Menores níveis de oxidação após o armazenamento refrigerado foram reportados em peças de carne (FASSEAS et al., 2008). Além de prolongar o prazo de validade da carne fresca, reporta-se a redução no crescimento de microorganismos durante 0 armazenamento refrigerado. Entretanto, sua inserção pode gerar um sabor muito forte aos produtos, resultando em baixa qualidade sensorial (CHOULIARA et al., 
2007). Sob condições de atmosfera modificada, tem sido reportado como retardador do crescimento de microorganismos deteriorantes (SKANDAMIS; NYCHAS, 2001).

\section{PRODUÇÃO DE COMPONENTES FUNCIONAIS DURANTE 0 PROCESSAMENTO}

\subsection{PRODUTOS CÁRNEOS SECOS CURADOS}

As carnes curadas a seco constituem produtos cárneos com sabor, cor e textura relevantes e particulares. Mudanças químicas e bioquímicas ocorridas durante 0 processamento e os mecanismos enzimáticos, são necessárias para formação do sabor, além da padronização da qualidade e desenvolvimento de composto bioativos (DEVINE; DIKEMAN, 2014). Um dos produtos curados estudados são os presuntos, sua qualidade nutricional é bastante variável e depende de muitos fatores intrínsecos do animal como também a composição da sua alimentação. O processo de cura a seco também afeta o teor de água, perdido durante a secagem, e o aumento no teor de sal, adicionado durante a etapa de salga (TOLDRÁ, 2016). Alguns compostos bioativos são disponibilizados através do processamento, entre eles os ácidos graxos e minerais. O presunto também contém dipeptídeos à base de histidina e taurina, glutamina, carnosina e anserina, com atividade antioxidante. Com base nas características específicas das dipeptidilpeptidases musculares, ambas as enzimas estão envolvidas principalmente na geração de peptídeos bioativos (DEVINE; DIKEMAN, 2014).

\subsection{REVESTIMENTO DE PRODUTOS CÁRNEOS}

A embalagem e revestimento de produtos cárneos esta ligado diretamente com a qualidade e a migração dos agentes da embalagem para o alimento. Reações de oxidação estão entre os fatores mais importantes responsáveis pela perda de qualidade em produtos cárneos. O uso de extratos vegetais naturais está se tornando uma alternativa importante no controle de mudanças oxidativas em produtos cárneos durante o armazenamento (ZHOU; XU; LIU, 2010). O uso de extratos de plantas é utilizado como revestimentos de produtos cárneos descritos na literatura, como Fan et al. (2019) que utlizou o extrato de Portulaca oleracea L. em carne suína e avaliou seu efeito antioxidante durante armazenamento refrigerado, obtendo resultado favorável ao uso do extrato. Em peito de frango fresco, Olaimat et al. (2014) pesquisou o efeito do óleo e extrato de mostarda oriental como revestimento para inibição de cepas Campylobacter jejuni, reduzindo o número viável de bactérias aeróbicas.

\subsection{FERMENTAÇÃO DE PRODUTOS CÁRNEOS}

Um produto de carne pode ser denominado "fermentado" se for preparado por um processo de amadurecimento em que os microrganismos desempenham um papel fundamental. Em cortes de carne crua e/ou salgada, atividade é restrita à superfície, enquanto o corte e moagem distribui e acrescentou microorganismos em todo o produto. Um número significativo de reações bioquímicas e físicas ocorre durante o processo de fermentação. Portanto, as características originais das matérias-primas são alteradas notavelmente, resultando em produtos com funcionalidade aprimorada (LÜCKE, 2015). A formação de metabólitos secundários através da fermentação esta presente no trabalho de $\mathrm{Ge}$ et al. (2019) que avaliou os efeitos do Lactobacillus plantarum NJAU-01 na oxidação proteica de salsichas fermentadas, no qual, concluiu-se potencial uma cultura com potencial antioxidante em embutidos fermentados. Chen et al. (2017) pesquisou o papel da fermentação bacteriana na lipólise e oxidação lipídica em salsichas secas de 
Harbin no desenvolvimento de sabor, concluiu-se que a fermentação bacteriana consegue inibir a oxidação lipídica, devido sua ação antioxidante.

\section{$4 \quad$ PRODUTOS CÁRNEOS INDUSTRIALIZADOS FUNCIONAIS}

\subsection{SALSICHA}

Salsicha é o produto cárneo industrializado, obtido da emulsão de carne de uma ou mais espécies de animais de açougue, adicionados de ingredientes, embutido em envoltório natural, ou artificial ou por processo de extrusão, e submetido a um processo térmico adequado (BRASIL, 2000). Foram desenvolvidas diversas pesquisas adicionando extratos de vegetais em suas formulações, para aumento de compostos bioativos e melhoria da qualidade. Yim et al. (2019) analisou os efeitos de extrato Caesalpinia sapan L. na estabilidade de cor, atividade antioxidante e antimicrobiana em salsichas de porco cozidas durante o armazenamento refrigerado, Almeida et al. (2015) analisou o efeito do extrato de casca de jabuticaba sobre a oxidação lipídica, a estabilidade microbiana e as propriedades sensoriais de salsichas do tipo Bolonha durante o armazenamento refrigerado.

\subsection{LINGUIÇA}

Entende-se por linguiça o produto cárneo industrializado, obtido de carnes de animais de açougue, adicionados ou não de tecidos adiposos, ingredientes, embutido em envoltório natural ou artificial, e submetido ao processo tecnológico adequado (BRASIL, 2000). Pesquisas realizadas avaliaram a influência de diferentes fontes de proteínas vegetais, de soro e de microalgas sobre as propriedades físico-químicas e perfil de aminoácidos de linguiças suínas frescas (MARTI-QUIJAL et al., 2019). Sebranek et al. (2005) comparou o extrato natural de alecrim e BHA/BHT para eficácia antioxidante relativa em linguiça de porco.

\subsection{MORTADELA}

Entende-se por Mortadela, o produto cárneo industrializado, obtido de uma emulsão das carnes de animais de açougue, acrescido ou não de toucinho, adicionado de ingredientes, embutido em envoltório natural ou artificial, em diferentes formas, e submetido ao tratamento térmico adequado. Carnes de diferentes espécies de animais de açougue, carnes mecanicamente separadas, até o limite máximo de 60\%; miúdos comestíveis de diferentes espécies de animais de açougue pele e tendões no limite máximo de 10\% e gorduras (BRASIL, 2000). Doménech-Asens et al. (2013) investigou o efeito da adição de extrato de tomate sobre as propriedades nutricionais e sensoriais da mortadela, Viuda-Martos et al. (2010) analisou o efeito da adição de óleos essenciais de fibra cítrica e especiarias sobre as características de qualidade e prazo de validade de mortadela.

\subsection{PRESUNTO COZIDO}

Presunto Cozido é o produto cárneo industrializado obtido exclusivamente com o pernil de suínos, desossado, adicionado de ingredientes, e submetido a um processo de cozimento adequado (BRASIL, 2000). Pesquisas mostram interesses nesse produto, principalmente na substituição de ingredientes, para enriquecimento nutricional. Pancrazio et al. (2016) estudou o extrato de levedura de cerveja usado como ingrediente em presuntos cozidos, outra linha de estudo são os compostos bioativos durante seu 
processamento, como Zhu et al. (2014) que pesquisou a estabilidade de um peptídeo antioxidante extraído do presunto.

\subsection{HAMBÚRGUER}

Hambúrguer é o produto cárneo industrializado obtido da carne moída dos animais de açougue, adicionado ou não de tecido adiposo e ingredientes, moldado e submetido a processo tecnológico adequado (BRASIL, 2000). Özvural et al. (2016) comparou a qualidade e características microbiológicas de hambúrgueres enriquecidos com extrato de chá verde utilizando três técnicas: adição direta, revestimento comestível e encapsulamento, Fernandes et al. (2016) pesquisou os efeitos do extrato de orégano na estabilidade oxidativa, microbiológica e sensorial de hambúrgueres de ovinos embalados em atmosfera modificada.

\section{CONSIDERAÇÕES FINAIS}

Os produtos cárneos têm um grande potencial para fornecer nutrientes na dieta. A composição nutricional destes, pode ser alterada pela adição direta de ingredientes alimentares bioativos ou a inclusão de compostos bioativos em dietas animais tendo a vantagem de que os compostos bioativos seriam biologicamente introduzidos na comida e, portanto, não teria que ser declarado como um aditivo alimentar. No entanto, apenas um número limitado de estudos sobre o possível benefício da carne funcional e produtos cárneos em humanos tem sido feito. A maioria das conclusões é tirada do fato de que os ingredientes em si podem ser benéficos para o ser humano. Portanto, são necessários maiores estudos que forneçam evidências benéficas para a saúde humana sobre a carne funcional e os produtos cárneos. Com o aumento dos dados científicos, pesquisadores e a indústria requerem maiores esforços em informar e educar os consumidores sobre os benefícios para a saúde proveniente destes produtos e, adicionalmente, garantirem a biodisponibilidade de ingredientes funcionais acrescidos, durante o processamento e armazenamento comercial.

\section{AGRADECIMENTOS}

Os autores agradecem a Coordenação de Aperfeiçoamento de Pessoal Superior (CAPES), Fundação Araucária e Conselho Nacional de Desenvolvimento Científico e Tecnológico (CNPq) pela assistência financeira.

\section{REFERÊNCIAS}

ALMEIDA, P. L.; DE LIMA, S. N.; COSTA, L. L.; DE OLIVEIRA, C. C.; DAMASCENO, K. A.; DOS SANTOS, B. A.; CAMPAGNOL, P. C. Effect of jabuticaba peel extract on lipid oxidation, microbial stability and sensory properties of Bologna-type sausages during refrigerated storage. Meat Science, v. 110, p.9-14, 2015.

ARIHARA, K. Functional foods. In: W. K. Jensen, C. Devine, \& M. Dikeman (Eds.), Encyclopedia of meat sciences, p.492-499. Oxford: Elsevier. 2004.

BAJPAI, V. K.; RAHMAN, A.; DUNG, N. T.; HUH, M. K.; KANG, S. C. In vitro inhibition of food spoilage and food borne pathogenic bacteria by essential oil and leaf extracts of Magnolia lilifloraDesr. Journal of Food Science, v. 73, p.314-320, 2008.

BIESALSKI, H. K. Meat as a component of a healthy diet - Are there any risks or benefits if meat is avoided in the diet? Meat Science, v. 70, p.509-524, 2005. 
BRASIL (2000). Secretaria de Defesa Agropecuária do Ministério da Agricultura e do Abastecimento. Regulamentos Técnicos de Identidade e Qualidade de Carne Mecanicamente Separada, de Mortadela, de Lingüiça e de Salsicha. Instrução Normativa n.4 de 31 de março de 2000.

BRASIL (2000). Secretaria de Defesa Agropecuária do Ministério da Agricultura e do Abastecimento. Regulamentos Técnicos de Identidade e Qualidade de Almôndega, de Apresuntado, de Fiambre, de Hamburguer, de Kibe, de Presunto Cozido e de Presunto. Instrução Normativa n.20 de 31 de julho de 2000.

CARNAGEY, K. M.; HUFF-LONERGAN, E. J.; TRENKLE, A.; WERTZ-LUTZ, A. E.; HORST, R. L.; BEITZ, D. C. Use of 25-hydroxyvitamin D3 and vitamin E to improve tenderness of beef form longissimus dorsi of heifers. Journal of Animal Science, v. 86, p.1649-1657, 2008.

CAVESTANY, M.; JIMENEZ, C. G.; SOLAS, M. T.; CARBALLO, J. Incorporation of sardine surimi to bologna sausage containing different fat levels. Meat Science, v. 38, p.27-37, 1994.

CHEN, Q.; KONG, B.; HAN, Q.; XIA, X.; XU, LI. The role of bacterial fermentation in lipolysis and lipid oxidation in Harbin dry sausages and its flavour development. LWT, v. 77, p.389-396, 2017.

CHIN, K. B.; KEETON, J. T.; MILLER, R. K.; LONGNECKER, M. T.; LAMKEY, J. W. Evaluation of konjac blends and soy protein isolate as fat replacements in low-fat bologna. Journal of Food Science, v. 65, p.756-763, 2000.

CHOULIARA, E.; KARATAPANIS, A.; SAVVAIDIS, I. N.; KONTOMINAS, M. G. Combined effect of oregano essential oil and modified atmosphere packaging on shelf-life extension of fresh chicken breast meat stored at $4^{\circ} \mathrm{C}$. Food Microbiology, v. 24, p.607-617, 2007.

DAWIDOWICZ, A. L.; WIANOWSKA, D.; BARANIAK, B. The antioxidant properties of alcoholic extracts from Sambucus nigraL. (antioxidant properties of extracts). Lebensmittel-Wissenschaft und Technologic, v. 39, p.308-315, 2006.

DEVINE, C.; DIKEMAN, M. Encyclopedia of meat sciences. Elsevier, 2014.

DOMÉNECH-ASENSI, G. et al. Effect of the addition of tomato paste on the nutritional and sensory properties of mortadella. Meat science, v. 93, n. 2, p.213-219, 2013.

DU, M.; NAM, K. C.; HUR, S. J.; ISMAIL, H.; KIM, Y. H.; AHN, D. U. Quality characteristics of irradiated chicken breast rolls from broilers fed different levels of conjugated linoleic acid. Meat Science, v. 63, p.249-255, 2003.

EASTWOOD, M. A. The physiological effect of dietary fiber: An update. Annual Review of Nutrition, v. 12, p.19-35, 1992.

EGGERT, J. M.; BELURY, M. A.; KEMPA-STECZKO, A.; MILLS, S. E.; SCHINCKEL, A. $P$. Effects of conjugated linoleic acid on the belly firmness and fatty acid composition of genetically lean pigs. Journal of Animal Science, v. 79, p.2866-2872, 2001. 
FAN, X.; LIU, S.; LI, H.; HE, J.; FENG, J.; ZHANG, X.; YAN, H. Effects of Portulaca oleracea L. extract on lipid oxidation and color of pork meat during refrigerated storage. Meat science, v. 147, p. 82-90, 2019.

FASSEAS, M. K.; MOUNTZOURIS, K. C.; TARANTILIS, P. A.; POLISSIOU, M.; ZERVAS, G. Antioxidant activity in meat treated with oregano and sage essential oils. Food Chemistry, v. 106, p.1188-1194, 2008.

FERNANDES, R. P. P. et al. Effects of oregano extract on oxidative, microbiological and sensory stability of sheep burgers packed in modified atmosphere. Food Control, v. 63, p.65-75, 2016.

GE, Q.; CHEN, S.; LIU, R.; YANG B.; YU, H.; WU, M.; ZHANG, W.; ZHOU, G. Effects of Lactobacillus Plantarum NJAU-01 on the protein oxidation of fermented sausage. Food Chemistry, 2019.

GRIGELMO-MIGUEL, N.; ABADIAS-SEROS,M. I.; MARTIN-BELLOSO, O. Characterisation of low-fat high-dietary fibre frankfurters. Meat Science, v. 52, p.247-256, 1999.

HONGSPRABHAS, P.; BARBUT, S. Effect of pre-heated whey protein level and salt on texture development of poultry meat batters. Food Research International, v. 32, p.145-149, 1999.

JIMENEZ-COLMENERO, F.; CARBALLO, J.; COFRADES, S. Healthier meat and meat products: Their role as functional foods. Meat Science, v. 59, p.5-13, 2001.

KWAK, N. S.; JUKES, D. J. Functional foods. Part 1. The development of a regulatory concept. Food Control, v. 12, p.99-107, 2001.

LIMA JÚNIOR, D. M.; MONTEIRO, P. B. S.; RANGEL, A. H. N.; URBANO, S. A.; MACIEL, M. V. Alimentos funcionais de origem animal. Revista Verde, v.6, n.2, p.30-40, 2011.

LÜCKE, F.-K. Quality improvement and fermentation control in meat products. In: Advances in Fermented Foods and Beverages. Woodhead Publishing, p.357-376, 2015.

MAIDMENT, D. C. F.; DEMBNY, Z.; HARDING, C. A study into the antibiotic effect of garlic Allium sativum on Escherichia coli and Staphylococcus albus. Nutrition and Food Science, v. 4, p.170-172, 1999.

MARINS, R. M.; ARAUJO, I. S. DE; JACOB, S. C. A propaganda de alimentos: orientação, ou apenas estímulo de consumo? Ciência Saúde Coletiva, v.16, n.9, p.3873-3882, 2011.

MARTI-QUIJAL, F. J.; ZAMUZ, S.; TOMA, I.; GÓMEZ, B.; ROCHETTI, G.; LUCINI, L.; REMIZE, F.; BARBA, F. J.; LORENZO, J. M. Influence of different sources of vegetable, whey and microalgae proteins on the physicochemical properties and amino acid profile of fresh pork sausages. LWT, v. 110, p.316-323, 2019.

MCCARTHY, T. L.; KERRY, J. P.; KERRY, J. F.; LYNCH, P. B.; BUCKLEY, D. J. Assessment of the antioxidant potential of natural food and plant extracts in fresh and previously frozen pork patties. Meat Science, v. 57, p.177-184, 2001. 
MENRAD, K. Market and marketing of functional food in Europe. Journal of Food Engineering, v. 56, pp. 181-188, 2003.

MUELLER, A. S.; MUELLER, K.; WOLF, N. M.; PALLAUF, J. Selenium and diabetes: An enigma. Free Radical Research, v. 43, p.1029-1059, 2009.

OLAIMAT, A. N.; FANG, Y.; HOLLEY, R. A. Inhibition of Campylobacter jejuni on fresh chicken breasts by $\mathrm{k}$-carrageenan/chitosan-based coatings containing allyl isothiocyanate or deodorized oriental mustard extract. International journal of food microbiology, $v$. 187, p.77-82, 2014.

OLIVEIRA, K. A. M.; SANTOS-MENDONCA, R. C.; GOMIDE, L. A. M.; VANETTI, M. C. D. Aqueous garlic extract and microbiological quality of refrigerated poultry meat. Journal of Food Processing and Preservation, v. 29, p.98-108, 2005.

ÖZVURAL, E. B.; HUANG, Q.; CHIKINDAS, M. L. The comparison of quality and microbiological characteristic of hamburger patties enriched with green tea extract using three techniques: Direct addition, edible coating and encapsulation. LWT-Food Science and Technology, v. 68, p.385-390, 2016.

PANCRAZIO, G.; CUNHA, S. C.; PINHO, P. G.; LOUREIRO, M.; MEIRELES, S.; FERREIRA, I. M. P. L. V. O.; PINHO, O. Spent brewer's yeast extract as an ingredient in cooked hams. Meat science, v. 121, p.382-389, 2016.

PEREZ-GAGO, M. B.; KROCHTA, J. M. Denaturation time and temperature effects on solubility, tensile properties and oxygen permeability of whey protein edible films. Journal of Food Science, v. 66, p.705-710, 2001.

PORCELLA, M. I.; SANCHEZ, G.; VAUDAGNA, S. R.; ZANELLI, M. L.; DESCALZO, A. M.; MEICHTRI, L. H. Soy protein isolate added to vacuum-packaged chorizos: Effect on drip loss, quality characteristics and stability during refrigerated storage. Meat Science, v. 57, p.437-443, 2001.

PROSKY, L. What is fiber? Current controversies. Trend in Food Science and Technology, v. 10, p.271-275, 1999.

ROTHSTEIN, W. G. Dietary fat, coronary heart disease, and cancer: A historical review. Preventive Medicine, v. 43, n. 5, p.356-360, 2006.

SEBRANEK, J. G.; SEWALT, V. J. H.; ROBBINS, K. L.; HOUSER, T. A. Comparison of a natural rosemary extract and $\mathrm{BHA} / \mathrm{BHT}$ for relative antioxidant effectiveness in pork sausage. Meat Science, v. 69, pp. 289-296, 2004.

SKANDAMIS, P. N.; NYCHAS, G. J. E. Effect of oregano essential oil on microbiological and physico-chemical attributes of minced meat stored in air and modified atmospheres. Journal of Applied Microbiology, v. 91, p.1011-1022, 2001.

TANABE, H.; YOSHIDA, M.; TOMITA, N. Comparison of the antioxidant activities of 22 commonly used culinary herbs and spices on the lipid oxidation of pork meat. Animal Science Journal, v. 73, p.389-393, 2002. 
TOLDRÁ, F. Dry-Cured Meats. Reference Module in Food Science, 2016.

VEKIARI, S. A.; OREOPOULOU, V.; TZIA, C.; THOMOPOULOS, C. D. Oregano flavonoids as lipid antioxidants. Journal of American Oil Chemistry Society, v. 70, p.483-487, 1993.

VERCRUYSSE, L.; VAN CAMP, J.; SMAGGHE, G. J. ACE Inhibitory peptides derived from enzymatic hydrolysates of animal muscle protein: A review. Journal of Agricultural and Food Chemistry, v. 53, p.8106-8115, 2005.

VIUDA-MARTOS, M.; NAVAJAS, R.; FERNANDEZ-LÓPEZ, J.; PÉREZ-ALVAREZ, J. A. Effect of added citrus fibre and spice essential oils on quality characteristics and shelf-life of mortadella. Meat Science, v. 85, n. 3, p.568-576, 2010.

WIEGAND, B. R.; PARRISH, F. C.; JR. SWAN, J. E.; LARSEN, S. T.; BASS, T. J. Conjugated linoleic acid improves feed efficiency, decreases subcutaneous fat, and improves certain aspects of meat quality in stress-genotype pigs. Journal of Animal Science, v. 79, p.2187-2195, 2002.

YETIM, H.; MULLER, W. D.; EBER, M. Using fluid whey in comminuted meat products: effects on technological, chemical and sensory properties of frankfutertype sausages. Food Research International, v. 34, p.97-101, 2001.

YIM, D. G.; SEO, J. K.; YUM, H. W.; ASHRAFUZZAMAN, M.; JOVEM, J. P.; PARVIN, R.; VAI, J. JIN, S. K.; KOO, O. K.; YANG, H. S. Effects of Caesalpinia sappan L. extract on the color stability, antioxidant and antimicrobial activity in cooked pork sausages during cold storage. LWT, v.112, p.1-6, 2019.

YU, L.; SCANLIN, L.; WILSON, J.; SCHMIDT, G. Rosemary extracts as inhibitors of lipid oxidation and color change in cooked turkey products during refrigerated storage. Journal of Food Science, v. 67, p.582-585, 2002.

ZHOU, G. H.; XU, X. L.; LIU, Y. Preservation technologies for fresh meat-A review. Meat science, v. 86, n. 1, p.119-128, 2010.

ZHU, C. Z.; ZHANG, W. G.; KANG, Z. L.; ZHOU, G. H.; XU, X. L. Stability of an antioxidant peptide extracted from Jinhua ham. Meat science, v. 96, n. 2, p.783-789, 2014. 